\title{
Adiponectin, Retinoic Acid Receptor Responder 2, and Peroxisome Proliferator-Activated Receptor- $\gamma$ Coativator-1 Genes and the Risk for Obesity
}

\author{
Ana Carolina Proença da Fonseca, ${ }^{1}$ Alan Cleveland Ochioni, ${ }^{1}$ Raisa da Silva Martins, ${ }^{2}$ \\ Verônica Marques Zembrzuski, ${ }^{1}$ Mario Campos Junior, ${ }^{1}$ Vivianne Galante Ramos, \\ João Regis Ivar Carneiro, ${ }^{3}$ José Firmino Nogueira Neto, ${ }^{4}$ Pedro Hernan Cabello, ${ }^{1,2}$ and \\ Giselda Maria Kalil Cabello ${ }^{1}$
}

${ }^{1}$ Human Genetic Laboratory, Oswaldo Cruz Institute/FIOCRUZ, Rio de Janeiro, RJ, Brazil

${ }^{2}$ Human Genetic Laboratory, Grande Rio University, Rio de Janeiro, RJ, Brazil

${ }^{3}$ Clementino Fraga Filho University Hospital, Rio de Janeiro, RJ, Brazil

${ }^{4}$ Department of Pathology and Laboratory, Rio de Janeiro State University, Rio de Janeiro, RJ, Brazil

Correspondence should be addressed to Giselda Maria Kalil Cabello; gkalil.fiocruz@gmail.com

Received 13 April 2017; Accepted 31 July 2017; Published 29 August 2017

Academic Editor: Mariann Harangi

Copyright (c) 2017 Ana Carolina Proença da Fonseca et al. This is an open access article distributed under the Creative Commons Attribution License, which permits unrestricted use, distribution, and reproduction in any medium, provided the original work is properly cited.

\begin{abstract}
Obesity is the most common nutritional disorder. This disease is a multifactorial disease influenced by environmental and genetic factors. This study investigated the relationship between common variants of adiponectin (ADIPOQ), retinoic acid receptor responder 2 (RARRES2), and peroxisome proliferator-activated receptor- $\gamma$ coativator-1 (PPARGC1) and obesity-related traits and susceptibility. A total of 167 individuals with obesity and 165 normal-weight subjects were recruited. Genotype frequencies of rs182052 in ADIPOQ differed significantly between the groups. Genotype AA was observed at a higher frequency in case than in control subjects. Association analysis showed that the A allele was a risk factor for obesity. This polymorphism was associated with body weight, body mass index (BMI), and waist circumference. After stratification by BMI, eutrophic individuals with AA or AG genotypes had higher body weights and waist circumferences than those with GG genotypes. In the case group, no associations were observed, except for stratified subjects with morbid obesity that exhibited a progressive increase of body weight, BMI, and waist circumference when rs182052 A was present. No associations were observed between SNPs in RARRES2 and PPARGC1 and obesity or any other studied variables. The rs182052 polymorphism in ADIPOQ is associated with a higher risk for obesity and obesity-related parameters.
\end{abstract}

\section{Introduction}

Obesity is a major public health problem worldwide [1]. This disease has an increasing prevalence in most countries, affecting populations from developed and developing countries (Figure 1). Recent data from the World Health Organization estimated that the prevalence of obesity more than doubled between 1980 and 2014 in the world. Currently, more than 1.9 billion adults have overweight and at least
600 million have obesity [2]. In Brazil, excess body weight affects half of all men and women, and data show that $20 \%$ of individuals have obesity [2,3]. Furthermore, the worldwide prevalence of obesity continues to increase at an alarming rate, resulting in nearly 3 million deaths every year [4].

In recent years, the population's weight gain has been influenced heavily by changes in eating and lifestyle that have increased the consumption of hypercaloric foods and reduced energy expenditure [5]. Even today, obesity 


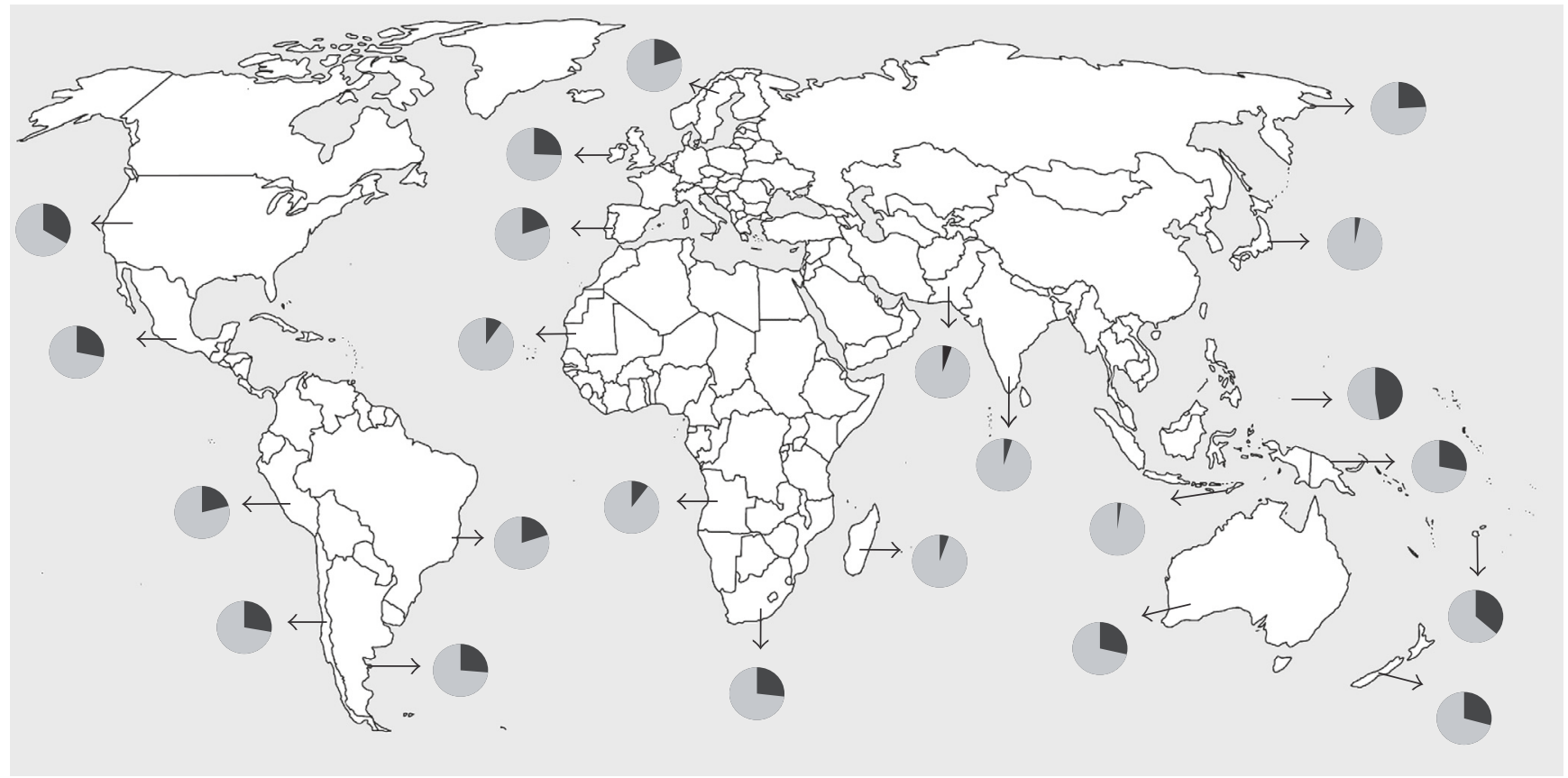

Prevalence:

Nonobese $(\mathrm{BMI}<30.0)$

Obese (BMI $\geq 30.0)$

Figure 1: Prevalence of obesity. Source: World Health Organization 2015.

is commonly portrayed stereotypically as a problem of gluttonous behavior. However, there are clear differences in susceptibility among different individuals or communities. This observation suggests that the control of body weight has a multifactorial nature and that genetic differences between individuals could play an important role in obesity risk within a given environment $[6,7]$.

Over the last two decades, researchers have made a continuous effort to identify genes and variants that predispose individuals to common forms of obesity. Over 120 candidate genes have been identified. However, less than $20 \%$ have been replicated by five or more other studies [8]. Among the confirmed genes are adiponectin (ADIPOQ), retinoic acid receptor responder 2 (RARRES2), and peroxisome proliferator-activated receptor- $\gamma$ coativator-1 (PPARGC1).

$A D I P O Q$ encodes adiponectin, a major adipocyte secretory protein. This protein acts in numerous tissues, regulating lipid and glucose metabolism, fat oxidation in the skeletal muscle and liver, and decreasing hepatic glucose production $[9,10]$. RARRES2 encodes the adipokine chemerin that reportedly plays a role in adipogenesis and adipocyte metabolism [11]. PPARGC1 is a member of a transcriptional coactivator family [12]. This coactivator protein regulates adaptive thermogenesis, mitochondrial fatty acid oxidation, hepatic gluconeogenesis, and adipogenesis, as well as glucose uptake and lipid metabolism [13-15].

These genes are responsible for encoding adipokines involved in the regulation of various metabolic pathways and are important for maintaining body energy homeostasis. Therefore, genetic variations may affect protein functions or gene expression efficiency and contribute to several pathophysiological conditions. The aim of the present study was to test the impact of common genetic variations in $A D I$ POQ, RARRES2, and PPARGC1 on obesity-related traits and susceptibility in a sample of adult subjects from Rio de Janeiro, Brazil.

\section{Materials and Methods}

2.1. Subjects. This case-control cross-sectional study comprised 332 adult subjects, aged 18 to 65 years, from the state of Rio de Janeiro in southeastern Brazil. Exclusion criteria were pregnancy, lactation, and the use of medication to lose weight. For this study, 167 individuals with obesity $(\mathrm{BMI} \geq 30.0)$ and 165 subjects with normal weight $(18.5 \leq$ $\mathrm{BMI} \leq 24.9)$ were selected. All people with obesity were recruited from a nongovernmental organization, the Rescue Group to Self-Esteem and Citizenship of the Obese (GRACO). The controls were volunteers from public hospitals of Rio de Janeiro, Brazil. The subject characteristics (anthropometric and biological) are shown in Table 1. All participants provided written consent to participate in this study, and the protocol was performed according to the Declaration of Helsinki (1964) and approved by Ethics Committee of the Oswaldo Cruz Foundation.

Anthropometrical characteristics, including height, weight, and waist and hip circumferences, were measured with a graduated tape by trained personal. We have estimated the pattern of fat distribution by dividing waist 
TABLE 1: Subject characteristics.

\begin{tabular}{|c|c|c|c|c|}
\hline & All & $\begin{array}{c}\text { Control } \\
(18.5 \leq \mathrm{BMI} \leq 24.9)\end{array}$ & $\begin{array}{c}\text { Case } \\
(\mathrm{BMI} \geq 30.0) \\
\end{array}$ & $P$ \\
\hline Age (years) & $35(27 ; 46)$ & $30(25 ; 38)$ & $40(31 ; 52)$ & $<0.001$ \\
\hline Gender (female/male) & $231 / 101$ & $98 / 67$ & $133 / 34$ & $<0.001$ \\
\hline Weight $(\mathrm{kg})$ & $82.2(63.0 ; 120.8)$ & $63.0(57.0 ; 70.3)$ & $120.0(102.4 ; 141.5)$ & $<0.001$ \\
\hline Height (m) & $1.65(1.60 ; 1.72)$ & $1.69(1.62 ; 1.74)$ & $1.63(1.58 ; 1.69)$ & $<0.001$ \\
\hline BMI $\left(\mathrm{kg} / \mathrm{m}^{2}\right)$ & $30.1(22.9 ; 45.3)$ & $22.8(21.0 ; 24.0)$ & $45.3(39.1 ; 51.6)$ & $<0.001$ \\
\hline Waist circumference $(\mathrm{cm})$ & $101.5(84.9 ; 131.3)$ & $85.0(76.5 ; 93.5)$ & $131.0(118.4 ; 144.5)$ & $<0.001$ \\
\hline Hip circumference $(\mathrm{cm})$ & $107.8(94.4 ; 140.0)$ & $94.5(84.0 ; 100.0)$ & $140.0(127.8 ; 151.0)$ & $<0.001$ \\
\hline WHR & $0.93(0.84 ; 1.02)$ & $0.87(0.80 ; 1.08)$ & $0.95(0.90 ; 1.01)$ & $<0.001$ \\
\hline WSWT & $144.8(130.5 ; 158.1)$ & $97.9(111.2 ; 129.0)$ & $144.8(130.5 ; 150.0)$ & $<0.001$ \\
\hline
\end{tabular}

Data are presented as median values (interquartile range) for continuous traits and $n(\%)$ for categorical traits. BMI: body mass index; WHR: waist-hip ratio; WSWT: waist circumference squared divided by weight. $P$ values for differences between case and control subjects.

circumference squared to weight (WSWT) which was adapted of Lutsey et al. [16].

2.2. Methods. Blood samples were collected from each participant, and genomic DNA was extracted using a commercial DNA extraction kit (QIAamp Blood Kit, Qiagen, Valencia, CA, USA). Genotypes for ADIPOQ (rs17366568 and rs182052), RARRES2 (rs17173608 and rs4721), and PPARGC1 (rs8192678 and rs3736265) variants were determined using real-time polymerase chain reaction TaqMan ${ }^{\circledR}$ assays (ThermoFisher, Carlsbad, CA, USA). Amplification was carried out in an ABI Prism 7500 Real-Time PCR System (ThermoFisher). All plates were included negative (all components excluding DNA) and positive internal controls for the genotyping quality conformation. There was $100 \%$ consistency in a $30 \%$ sample of duplicating test.

2.3. Statistical Analyses. Normality of continuous variables was tested by the Kolmogorov-Smirnov and Shapiro Wilk tests. Differences in continuous and categorical variables between two groups were calculated by the Mann-Whitney and $\chi^{2}$ tests, respectively.

Genotype and allele frequencies were estimated by gene counting. Genotype frequencies for each single nucleotide polymorphism (SNP) were tested for Hardy-Weinberg equilibrium using the $\chi^{2}$ test. Allele and genotype frequencies between control and case groups were compared by logistic regression, and the odds ratio (OR) was calculated. Differences on the genotype frequency between groups were carried out in dominant and recessive models. Logtransformation of anthropometric variables was performed before linear regression. The traits were chosen as the dependent variable, and the genotypes were tested as the independent variable (additive model). All regressions were adjusted for gender and age. The analyses were performed using the SPSS statistical package (IBM, Chicago, IL, USA). A $P$ value less than 0.05 was considered statistically significant.

Sample size was calculated using an iterative process to compute the sample needed to test the difference between the two groups of a qualitative variable [17]. A conservative and convenience sample was chosen, since different polymorphisms were analyzed in this study $(80 \%$ of statistical power).

\section{Results}

3.1. Basic Patient Characteristics. Anthropometric and biological variables of the 332 subjects, divided into cases and controls according to body mass index (BMI) status, are shown in Table 1. As expected, individuals with obesity had higher values of body measures than the control group. The exception was in height, which the control group had higher values.

3.2. Genotyping of Patient Samples Related to the Increased Risk for Obesity. In the present study, all six tested SNPs were polymorphic (minor allele frequency $>0.01$ ). The sample was stratified by BMI, and details about the genotype and allele frequencies are presented in Table 2. Only SNP rs17366568 was not in Hardy-Weinberg equilibrium, both for cases $(P<0.001)$ and controls $(P<0.001)$. Therefore, rs17366568 was excluded in the following analyses.

Genotype frequencies of rs182052 in ADIPOQ were differed between the groups. Genotype AA was associated with obesity when compared to GG $(P=0.014)$, even after adjusted for gender and age $(P=0.023)$. In order to explore this association, we carried out the dominant and recessive models. The rs182052 was significantly associated with obesity in both models. However, after adjustment, this polymorphism was only related to obesity in a recessive model $(P=0.049)$. Furthermore, the allelic test showed that the minor allele frequency (A) was significantly higher in subjects with obesity than in controls (38.9 versus $29.4 \%$ ). Therefore, individuals carrying the A allele had an increased risk for obesity $(\mathrm{OR}=1.53$ [1.11-2.11]). We did not find associations between SNPs in RARRES2 and PPARGC1 and obesity.

3.3. Associations between SNPs and Anthropometric Data. We investigated the impact of the SNPs on anthropometric variables. $A D I P O Q$ rs182052 was associated with body weight $(P=0.009)$, BMI $(P=0.022)$, waist circumference $(P=0.005)$, and WSWT values $(P=0.034)$, after adjusting 
TABLE 2: Genotype and allele frequencies of control and case individuals and association with the risk of obesity.

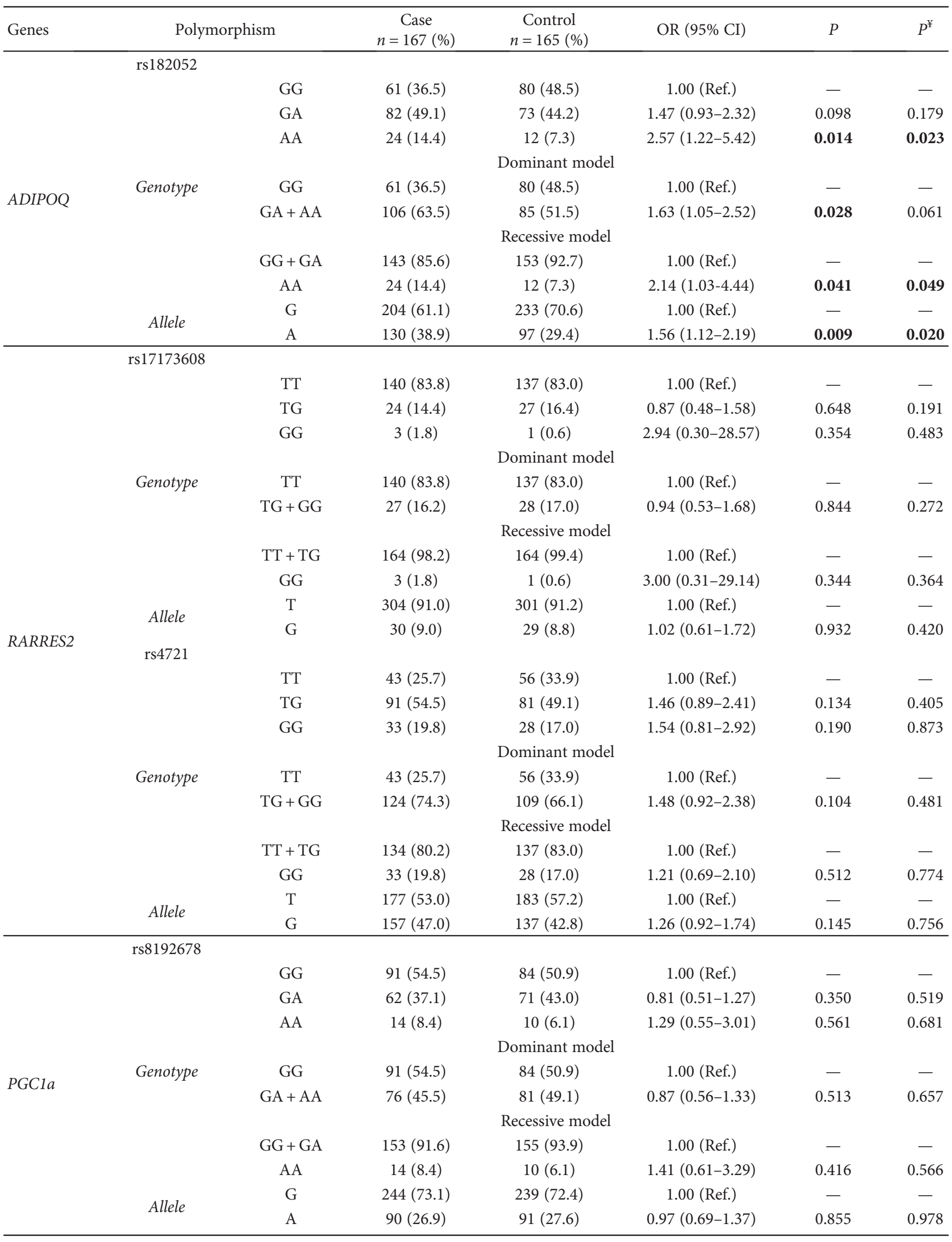


TABLE 2: Continued.

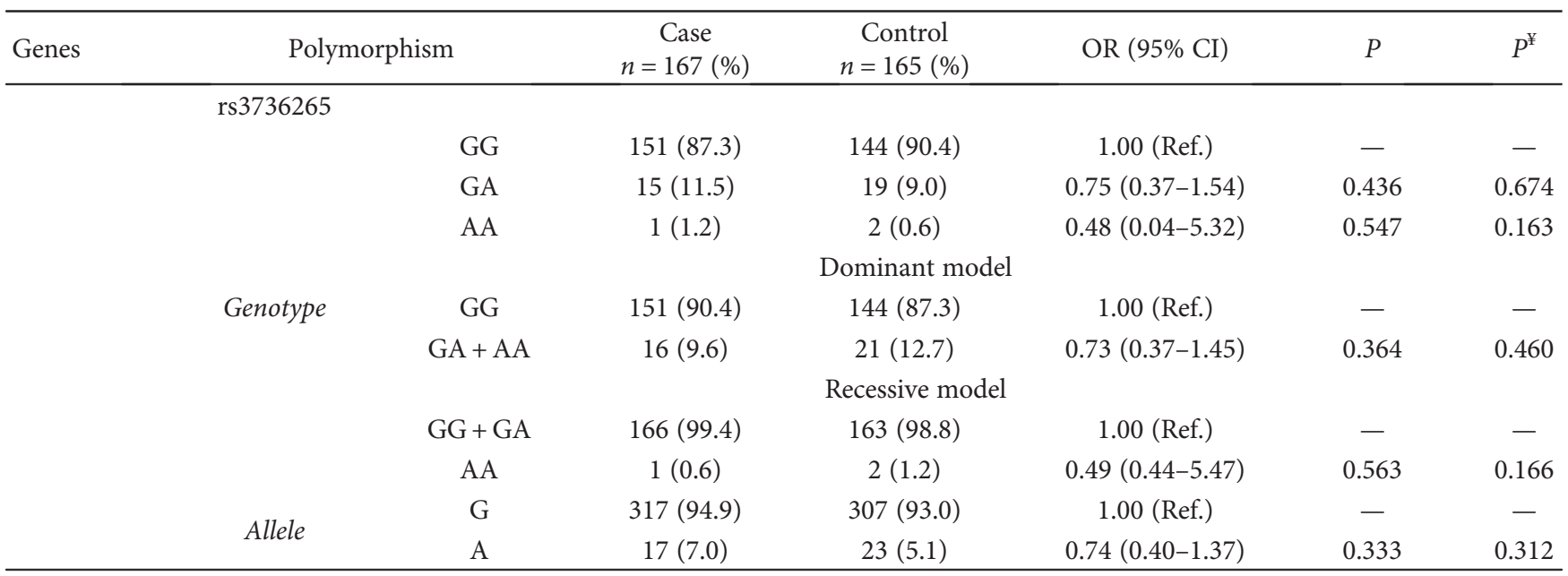

$P$ values for logistic regression. $P^{¥}$ values adjusted for gender and age.

TABLE 3: Correlations of $A D I P O Q$ rs182052 with anthropometric traits in the total patient sample.

\begin{tabular}{|c|c|c|c|c|}
\hline \multirow[b]{2}{*}{ Parameters } & \multicolumn{3}{|c|}{ ADIPOQ rs182052 } & \multirow[b]{2}{*}{$P$} \\
\hline & $\begin{array}{c}\text { GG } \\
(n=141)\end{array}$ & $\begin{array}{c}\mathrm{GA} \\
(n=155)\end{array}$ & $\begin{array}{c}\text { AA } \\
(n=36)\end{array}$ & \\
\hline$\overline{\text { Age (years) }}$ & $34.0(27.0 ; 42.0)$ & $36.0(28.0 ; 47.0)$ & $33.0(28.0 ; 50.0)$ & 0.323 \\
\hline Weight (kg) & $72.0(59.9 ; 116.4)$ & $83.0(65.2 ; 125.0)$ & $92.0(71.9 ; 127.2)$ & 0.009 \\
\hline BMI $\left(\mathrm{kg} / \mathrm{m}^{2}\right)$ & $24.7(22.06 ; 44.20)$ & $30.7(22.92 ; 46.72)$ & $34.9(24.3 ; 49.1)$ & 0.022 \\
\hline Waist circumference $(\mathrm{cm})$ & $98.0(81.0 ; 130.00)$ & $103.0(85.8 ; 133.5)$ & $111.8(96.0 ; 139.5)$ & 0.005 \\
\hline Hip circumference $(\mathrm{cm})$ & $102.0(92.3 ; 134.0)$ & $110.0(95.8 ; 142.0)$ & $124.0(100.9 ; 142.5)$ & 0.204 \\
\hline WHR & $0.92(0.82 ; 1.01)$ & $0.93(0.84 ; 1.02)$ & $0.94(0.89 ; 0.99)$ & 0.349 \\
\hline WSWT & $123.0(130.1 ; 147.3)$ & $133.0(112.4 ; 153.3)$ & $133.5(117.4 ; 158.0)$ & 0.034 \\
\hline
\end{tabular}

Data are present as median values (interquartile range). BMI: body mass index; WHR: waist-hip ratio; WSWT: waist circumference squared divided by weight. $P$ values for linear regression. Age was adjusted for gender. All obesity-related traits were adjusted for gender and age.

for gender and age (Table 3). Individuals carrying the A allele had higher body weight, BMI, waist circumference, and WSWT values than those homozygous for the G allele. None of the SNPs tested were associated with the waist-to-hip ratio (WHR).

Subsequently, the sample was stratified by BMI, and the analyses were made separately in the case and control groups. In the control subjects, the median body weight $(P=0.019)$ and waist circumference $(P=0.014)$ were different between the rs182052 genotype groups and the presence of the minor allele (A) was associated with higher progressive measurements (Table 4). In the case group, no association was found between any SNP and the anthropometric traits studied (data not shown).

Additionally, in individuals with morbid obesity $(\mathrm{BMI} \geq 40.0)$, associations were found between $A D I P O Q$ rs182052 and body weight $(P=0.006)$, BMI $(P=0.002)$, and waist circumference $(P=0.013)$ (Table 4$)$.

We did not find associations between SNPs in RARRES2 and PPARGC1, and anthropometric variables, either before or after stratification.

\section{Discussion}

In this study, ADIPOQ rs182052 was associated with obesity susceptibility. The AA genotype increased the risk for obesity when compared with genotype GG. Additional analysis showed that polymorphism is associated with obesity in recessive model. Moreover, our results showed that individuals carrying the A allele were more susceptible to obesity when compared to those with the wild-type allele. A previous study reported a similar result in Korean women, in which the A allele was associated with an increased risk for obesity [18].

There were some associations between SNPs and anthropometric characteristics. Our results showed a doseeffect relationship between ADIPOQ rs182052 and obesity measurements. We found that subjects with genotypes AA or AG had higher body weight, BMI, waist circumference, and WSWT values after adjustment for gender and age. Furthermore, when stratified by BMI, there were significantly higher values of body weight and waist circumference in control subjects carrying the A allele. In the entire group with 


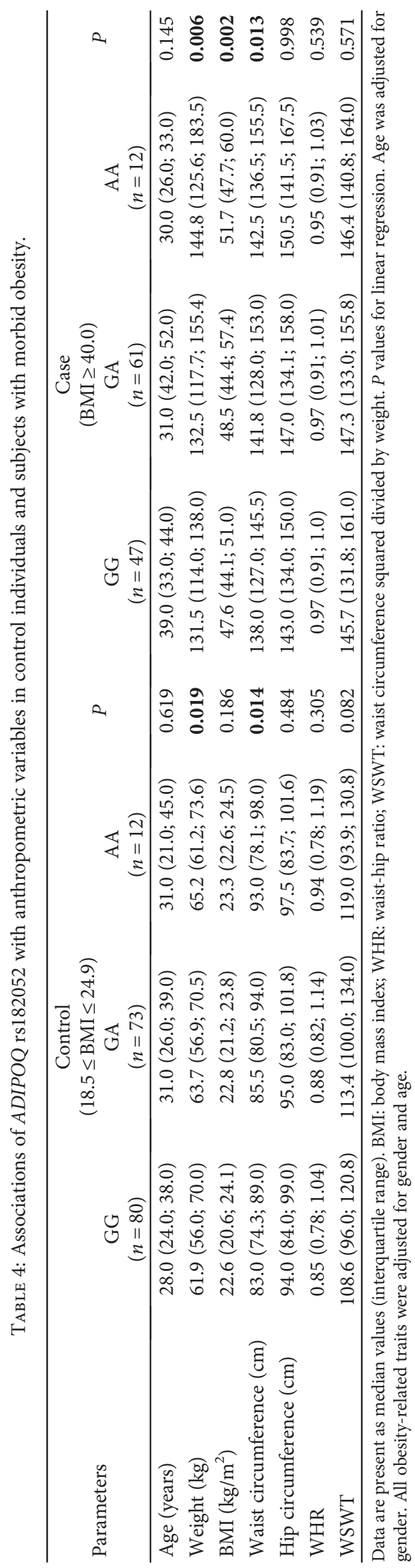


obesity, we failed to detect any associations between the variables tested. However, increased body weight, BMI, and waist circumference were found in individuals with morbid obesity carrying the same risk allele. These results showed that the rs182052 polymorphism was associated with anthropometric traits in control and individuals with morbid obesity, but not the entire sample with obese people. There is no clear explanation for this result. However, it may be due to the limited sample size of obesity class I $(30.0 \leq \mathrm{BMI} \leq 34.9)$ and $\mathrm{II}(35.0 \leq \mathrm{BMI} \leq 39.9)$ individuals.

Previous studies reported an association between rs182052 and obesity-related traits. Associations found in the current study are in agreement with Sutton et al. [19] who showed that the minor allele (A) was associated with higher BMI and waist circumference values in a Hispanic population. Similarly, Richardson et al. [20] found that the rs182052 A allele in Mexican individuals was significantly associated with an increased BMI. In the studies of Korean inhabitants, the same allele was related to increased body weight, WHR, and BMI in women [18]. In contrast, we did not find an association between this polymorphism and WHR. Interestingly, in a study on a cohort of Caucasian Americans, researchers found an interaction between rs182052, waist circumference, and levels of adiponectin. The Caucasian subjects with genotype AA had a reduction of $15.6 \%$ of serum adiponectin and an increased waist circumference when compared with those of genotype GG [21]. In another study, Hivert et al. [22] did not find an association between this polymorphism and BMI and waist circumference in a large cohort of European descent. In addition, a family-based study from a genetically isolated rural population of the Netherlands did not show a relationship between rs182052 and BMI or waist circumference [23]. These conflicting results may be attributable to differences in genetic background and different sample criteria, or other environmental factors [24].

The contribution of adiponectin to obesity is not well understood, but some studies have shown that individuals with obesity have lower plasma concentration of this protein than subjects without obesity $[25,26]$. In addition, ADIPOQ is very polymorphic and several SNPs are associated with adiponectin levels [22, 27-29]. The rs182052 SNP is located in the first intron of $A D I P O Q$. However, the first intron of human $A D I P O Q$ also contains a gene expression enhancer element, responsible for increasing the activity of its promoter [30]. Variants in this promoter region affect circulating adiponectin levels by modifying ADIPOQ expression. Thus, hypoadiponectinemia has been observed consistently with obesity $[25,26]$. Based on these findings, we suggest that the ADIPOQ rs182052 polymorphism may be affecting adiponectin serum levels in our subjects and may disrupt energy balance by reducing energy expenditure [31]. This disruption, associated with an obesogenic environment, could result in increased body adiposity. However, replication and validation studies are necessary to confirm the role of $A D I P O Q$ in the genetic susceptibility to obesity in different populations.

In this study, we have used a well-characterized cohort of participants, with or without obesity. Our sample has a significant portion of morbidly obese subjects, which are more difficult to recruit. However, one limitation of this study came from the cross-sectional study design, since we are not able to consider the fluctuation of body weight and other measurements over time. Furthermore, we are not able to fully isolate environmental factors, which could have an influence on the outcome.

In conclusion, our case-control study made in a southeastern Brazilian population suggests that the rs 182052 polymorphism of $A D I P O Q$, but not the other SNPs tested, is associated with an increased risk for obesity and obesityrelated parameters.

\section{Conflicts of Interest}

The authors declare that there is no conflict of interest regarding the publication of this paper.

\section{Acknowledgments}

The authors thank Láiza Almeida Cabral for her great technical assistance and Rosimere Lima for her excellent work with patients in GRACO. Furthermore, they are grateful to the patients and healthy volunteers who kindly agreed to participate in this study. This work was supported by the Oswaldo Cruz Foundation (FIOCRUZ, Brazil) and National Council for Scientific and Technological Development (CNPq). The funding source had no involvement in the conduct of the research and/or preparation of the article.

\section{References}

[1] T. Huang and F. B. Hu, "Gene-environment interactions and obesity: recent developments and future directions," $B M C$ Medical Genomics, vol. 5, article S2, 2015.

[2] World Health Organization, "Obesity and overweight," 2016, October 2016, http://www.who.int/mediacentre/factsheets/ fs $311 /$ en/.

[3] R. Yakota, B. Iser, L. Sardinha, L. Moura, R. Claro, and D. Malta, "Short term trends of obesity using data of the telephone based surveillance system of risk and protective factors for chronic disease (VIGITEL), Brazil, 2006 to 2009," Journal of Epidemiology Community Health, vol. 65, article A315, 2011.

[4] M. M. Finucane, G. A. Stevens, M. J. Cowan et al., "National, regional, and global trends in body-mass index since 1980: systematic analysis of health examination surveys and epidemiological studies with 960 country-years and 9.1 million participants," Lancet, vol. 377, pp. 557-567, 2011.

[5] C. P. Rodrigo, "Current mapping of obesity," Nutricion Hospitalaria, vol. 28, pp. 21-31, 2013.

[6] Q. Xia and S. F. Grant, "The genetics of human obesity," Annual of the New York Academic Science, vol. 1281, pp. 178-190, 2013.

[7] J. S. El-Sayed Moustafa and P. Froguel, "From obesity genetics to the future of personalized obesity therapy," Nature Reviews Endocrinology, vol. 9, pp. 402-413, 2013.

[8] T. Rankinen, A. Zuberi, Y. C. Chagnon et al., "The human obesity gene map: the 2005 update," Obesity, vol. 14, pp. 529-644, 2006. 
[9] K. Rabe, M. Lehrke, K. G. Parhofer, and U. C. Broedl, "Adipokines and insulin resistance," Molecular Medicine, vol. 14, pp. 741-751, 2008.

[10] M. J. Khandekar, P. Cohen, and B. M. Spiegelman, "Molecular mechanisms of cancer development in obesity," Nature Reviews Cancer, vol. 11, pp. 886-895, 2011.

[11] K. B. Goralski, T. C. McCarthy, E. Hanniman et al., "Chemerin, a novel adipokine that regulates adipogenesis and adipocyte metabolism," Journal of Biological Chemistry, vol. 282, pp. 28175-28188, 2007.

[12] B. N. Finck, M. C. Gropler, Z. Chen et al., "Lipin 1 is an inducible amplifier of the hepatic PGC-1alpha/PPARalpha regulatory pathway," Cell Metabolism, vol. 4, pp. 199-210, 2006.

[13] P. Puigserver and B. M. Spiegelman, "Peroxisome proliferatoractivated receptor-gamma coactivator 1 alpha (PGC-1 alpha): transcriptional coactivator and metabolic regulator," Endocrine Reviews, vol. 24, pp. 78-90, 2003.

[14] T. Kunej, M. Globocnik Petrovic, P. Dovc, B. Peterlin, and D. Petrovic, "A Gly482Ser polymorphism of the peroxisome proliferator-activated receptor- $\gamma$ coactivator-1 (PGC-1) gene is associated with type 2 diabetes in Caucasians," Folia Biologica, vol. 50, pp. 157-158, 2004.

[15] G. Medina-Gomez, S. Gray, and A. Vidal-Puig, "Adipogenesis and lipotoxicity: role of peroxisome proliferator-activated receptor gamma (PPAR $\gamma)$ and PPAR $\gamma$ coactivator-1 (PGC1)," Public Health Nutrition, vol. 10, pp. 1132-1137, 2007.

[16] P. L. Lutsey, M. A. Pereira, A. G. Bertoni, N. R. Kandula, and R. J. Jacobs David, "Interactions between race/ethnicity and anthropometry in risk of incident diabetes: the multiethnic study of atherosclerosis," American Journal of Epidemiology, vol. 172, no. 2, pp. 197-204, 2010.

[17] J. H. Jar, Bioestatistical Analisis, Prentice-Hall, Inc, NJ: USA, 4 edition, 1999.

[18] M. Doo and Y. Kim, "Association between ADIPOQ gene polymorphism rs182052 and obesity in Korean women," Genomics \& Informatics, vol. 8, pp. 116-121, 2010.

[19] B. S. Sutton, S. Weinert, C. D. Langefeld et al., "Genetic analysis of adiponectin and obesity in Hispanic families: the IRAS family study," Human Genetics, vol. 117, pp. 107-118, 2005.

[20] D. K. Richardson, J. Schneider, M. J. Fourcaudot et al., "Association between variants in the genes for adiponectin and its receptors with insulin resistance syndrome (IRS)related phenotypes in Mexican Americans," Diabetologia, vol. 49, pp. 2317-2328, 2006.

[21] C. L. Wassel, J. S. Pankow, D. R. Jacobs, M. W. Steffes, N. Li, and P. J. Schreiner, "Variants in the adiponectin gene and serum adiponectin: the Coronary Artery Development in Young Adults (CARDIA) study," Obesity, vol. 18, pp. 23332338, 2010.

[22] M. Hivert, A. K. Manning, J. B. Mcateer et al., "Common variants in the adiponectin gene (ADIPOQ) associated with plasma adiponectin levels, type 2 diabetes, and diabetesrelated quantitative traits," Diabetes, vol. 57, pp. 3353-3359, 2008.

[23] P. Henneman, Y. Aulchenko, and R. Frants, "Genetic architecture of plasma adiponectin overlaps with the genetics of metabolic syndrome-related traits," Diabetes Care, vol. 33, pp. 908-913, 2010.

[24] J. Du, Q. Li, X. Yu et al., "Genetic variants in the ADIPOQ gene and the risk of metabolic syndrome: a case-control study of a
Chinese Han population," Annals of Human Genetics, vol. 76, no. 2, pp. 101-109, 2012.

[25] M. Valle, R. Martos, and R. M. Morales, "Low-grade systemic inflammation, hypoadiponectinemia and a high concentration of leptin are present in very young obese children, and correlate with metabolic syndrome," Diabetes and Metabolic Syndrome: Clinical Research \& Reviews, vol. 31, pp. 55-62, 2005.

[26] N. Kubota, W. Yano, T. Kubota et al., “Adiponectin stimulates AMP-activated protein kinase in the hypothalamus and increases food intake," Cell Metabolism, vol. 6, pp. 55-68, 2007.

[27] T. Kyriakou, L. J. Collins, N. J. Spencer-jones et al., “Adiponectin gene ADIPOQ SNP associations with serum adiponectin in two female populations and effects of SNPs on promoter activity," Journal of Human Genetics, vol. 53, pp. 718-727, 2008.

[28] J. F. Ferguson, C. Phillips, C. Tierney et al., "Gene-nutrient interactions in the metabolic syndrome: the role of adiponectin," Proceedings of the Nutrition Society, vol. 13, p. 67: E265, 2009.

[29] I. M. Heid, P. Henneman, A. Hicks et al., "Clear detection of ADIPOQ locus as the major gene for plasma adiponectin: results of genome-wide association analyses including 4659 European individuals," Atherosclerosis, vol. 208, pp. 412-420, 2011.

[30] L. Qiao, P. S. Maclean, J. Schaack et al., "C/EBP $\alpha$ regulates human adiponectin gene transcription through an intronic enhancer," Diabetes, vol. 54, pp. 1744-1754, 2005.

[31] Y. Arita, S. Kihara, N. Ouchi et al., "Paradoxical decrease of an adipose-specific protein, adiponectin, in obesity," Biochemical and Biophysical Research Communications, vol. 83, pp. 7983, 1999. 


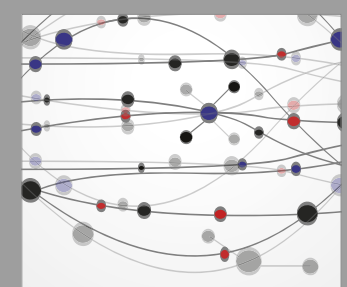

The Scientific World Journal
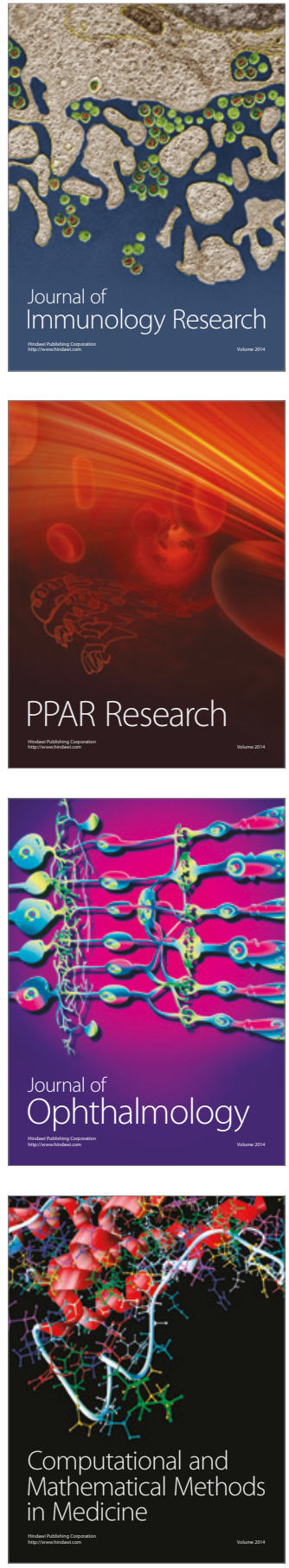

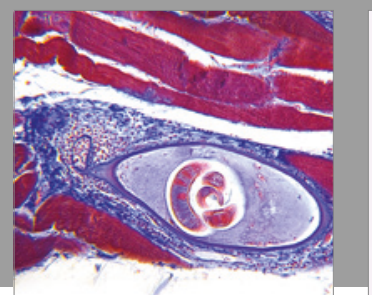

Gastroenterology Research and Practice
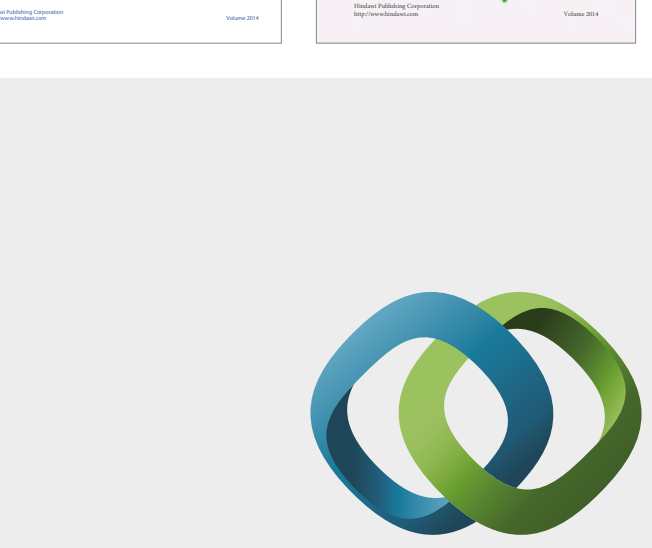

\section{Hindawi}

Submit your manuscripts at

https://www.hindawi.com
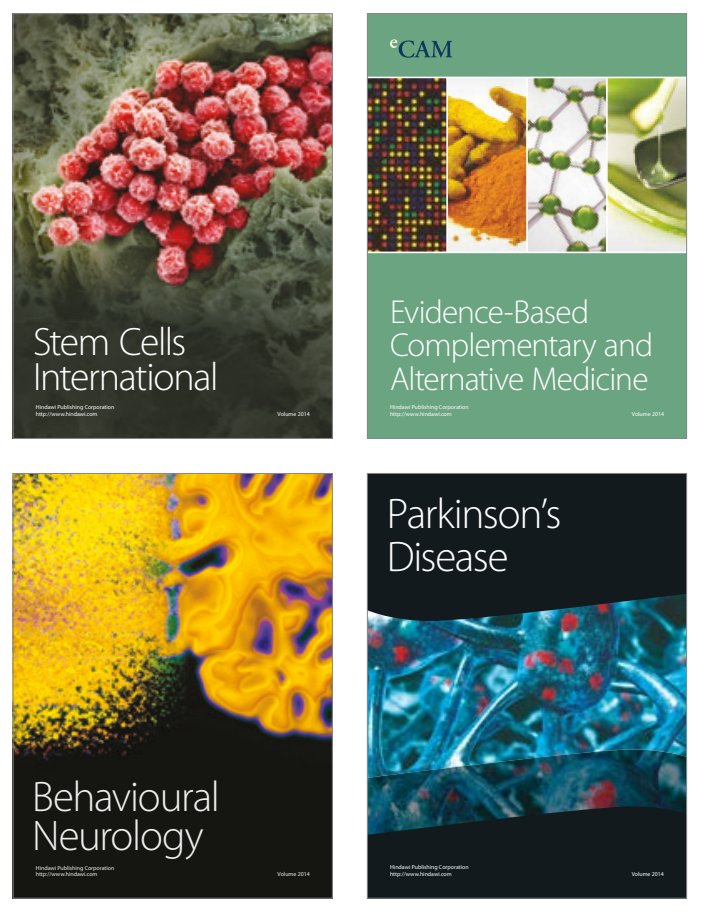
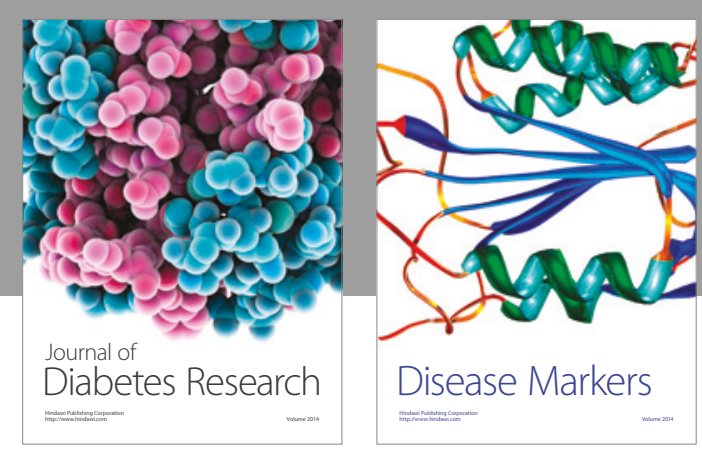

Disease Markers
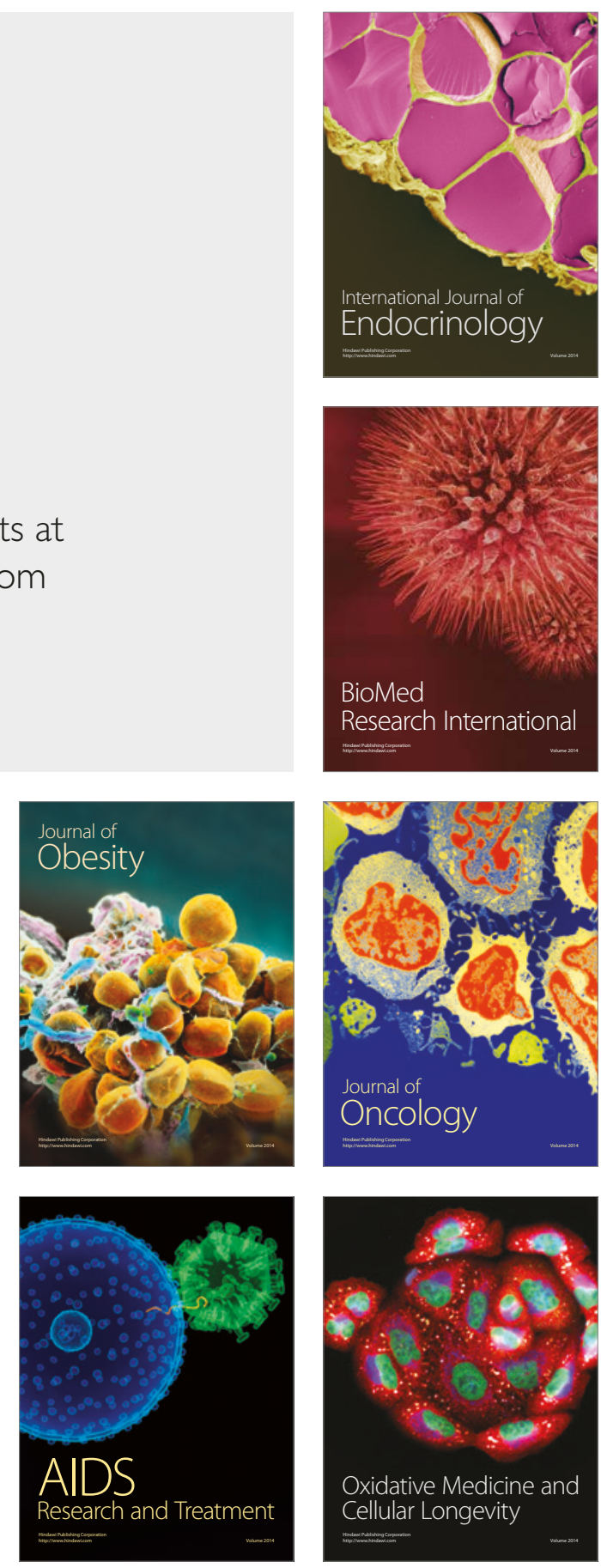